\title{
Asymptotic Solutions of Time-Space Fractional Coupled Systems by Residual Power Series Method
}

\author{
Wenjin $\mathrm{Li}^{1}$ and Yanni Pang ${ }^{2}$ \\ ${ }^{1}$ School of Applied Mathematics, Jilin University of Finance and Economics, Changchun, Jilin 130117, China \\ ${ }^{2}$ School of Mathematics, Jilin University, Changchun, Jilin 130012, China \\ Correspondence should be addressed to Yanni Pang; pangyn@jlu.edu.cn
}

Received 19 March 2017; Revised 15 May 2017; Accepted 30 May 2017; Published 10 July 2017

Academic Editor: Qasem M. Al-Mdallal

Copyright ( 2017 Wenjin Li and Yanni Pang. This is an open access article distributed under the Creative Commons Attribution License, which permits unrestricted use, distribution, and reproduction in any medium, provided the original work is properly cited.

This paper focuses on the asymptotic solutions to time-space fractional coupled systems, where the fractional derivative and integral are described in the sense of Caputo derivative and Riemann-Liouville integral. We introduce the Residual Power Series (for short RPS) method to construct the desired asymptotic solutions. Furthermore, we apply this method to some time-space fractional coupled systems. The simplicity and efficiency of RPS method are shown by the application.

\section{Introduction}

Fractional derivative was mentioned in a letter from L'Hopital to Leibniz in 1695. In the letter, L'Hopital proposed a question "What is the result of $d^{n} y / d x^{n}$ if $n=1 / 2$ ?" The answer of Leibniz was " $d^{1 / 2} x$ will be equal to $x \sqrt{d x: x}$. This is an apparent paradox, from which, one day useful consequences will be drawn" $[1,2]$. Furthermore, the generalization of this framework indicates that it is more appropriate to talk of integration and differentiation of, such as fractional order, real number order and even complex number order just as the development of number system. However, there is a basic question: "What is fractional integral and derivative?" Or "How to define the fractional integral and derivative?" More and more mathematicians focused on this problem, such as J. L. Lagrange, P. S. Laplace, and Joseph B. J. Fourier. Some different definitions of fractional integrals and derivatives have been defined according to different needs, like Riemann-Liouville integral, Caputo derivative, Weyl derivative, and so on $[2,3]$. But there is no uniform definition of fractional integral and derivative, and the frequently used definition is Riemann-Liouville integral and Caputo derivative.

Fractional differential equations, which involve fractional order derivatives, are applied in many engineering and scientific disciplines as the mathematical modelling of systems and processes in the fields of physics, chemistry, aerodynamics, electrodynamics of complex medium, polymer rheology, and so on. Fractional differential equations also serve as an excellent tool for the description of hereditary properties of various materials and processes. An essential topic is to construct the solutions to fractional differential equations. And there are some effective methods to obtain different kinds of solutions, like Sumudu transform and variational iteration method [4], fractional Taylor vector approximate method [5], iterative method [6-8], Residual Power Series (RPS) method [9-13], and so on [14-16]. On the other hand, the study of coupled systems which involve fractional differential equations is also important because fractional coupled systems occur in many fields [17-21]. In this paper, we generalize the RPS method to time-space fractional coupled systems and obtain the asymptotic series solutions.

The organization of this paper is as follows: In Section 2, some concepts and lemmas on fractional calculus are presented. In Section 3, we introduce the algorithm of RPS method for time-space fractional coupled system. In Section 4, asymptotic solutions of some examples are solved via RPS method. In Section 5, some concluding remarks are presented. 


\section{Preliminaries}

In this section, some concepts and main lemmas we need in this paper are presented [2, 3, 22-24]. And more details about fractional calculus can be found in $[2,23,24]$.

Definition 1. A real function $f(x)$ is said to be in the space $C_{\mu}, \mu \in \mathbb{R}$ if there exists a real number $\rho>\mu$ such that $f(x)=$ $x^{\rho} f_{1}(x)$, where $f_{1}(x) \in C[0, \infty)$. And it is said to be in the space $C_{\mu}^{n}$ if $f^{(n)}(x) \in C_{\mu}, n \in \mathbb{N}$.

Definition 2. The Riemann-Liouville fractional integral operator of order $\alpha \geqslant 0$ of a function $f \in C_{\mu}, \mu \geqslant-1$ is defined as

$$
\begin{aligned}
& I_{t}^{\alpha} f(t) \\
& \quad:= \begin{cases}\frac{1}{\Gamma(\alpha)} \int_{0}^{t}(t-\tau)^{\alpha-1} f(\tau) \mathrm{d} \tau, & \alpha>0, t>\tau \geqslant 0, \\
f(t), & \alpha=0,\end{cases}
\end{aligned}
$$

where the symbol $I_{t}^{\alpha}$ represents the $\alpha$ th Riemann-Liouville fractional integral of $f$.

Definition 3. The Caputo fractional derivative of order $\alpha>0$ of $f \in C_{-1}^{n}, n \in \mathbb{N}$ is defined as

$$
D_{t}^{\alpha} f(t):= \begin{cases}I_{t}^{n-\alpha} f^{(n)}(t), & n-1<\alpha<n, t>0, \\ \frac{\mathrm{d}^{n} f(t)}{\mathrm{d} t^{n}}, & \alpha=n,\end{cases}
$$

where the symbol $D_{t}^{\alpha} f(t)$ represents the $\alpha$ th Caputo fractional derivative of $f$.

Definition 4. The power series

$$
\sum_{n=0}^{\infty} c_{n}\left(t-t_{0}\right)^{n \alpha}=c_{0}+c_{1}\left(t-t_{0}\right)+c_{2}\left(t-t_{0}\right)^{2}+\cdots
$$

is called a fractional power series about $t=t_{0}$, where $t$ is a variable and $c_{n}(n=0,1,2 \ldots)$ are the coefficients of the series, $\alpha \in \mathbb{R}^{+}$.

Remark 5. For convenience, we shall treat $t_{0}=0$. In fact, the transformation $\mathscr{T}: t^{\prime}=t-t_{0}$ reduces the fractional power series about $t=t_{0}$ to the fractional power series about $t=0$ and meanwhile the transformation $\mathscr{T}$ is reversible.

Definition 6. A function $f(t)$ is analytical at $t=0$ if $f(t)$ can be written as a form of fractional power series.

Lemma 7. Suppose that $f(t)$ is an analytic function at $t=0$; then $f(t)$ can be written as follows:

$$
f(t)=\sum_{n=0}^{\infty} c_{n} t^{n \alpha}, \quad 0<\alpha \leq 1,|t|<R
$$

Furthermore, if $f(t) \in C(-R, R)$ and $D_{t}^{n \alpha} f(t) \in C(-R, R)$ for $n=0,1,2 \ldots$ then the coefficients $c_{n}$ will take the form

$$
c_{n}=\frac{\left.D_{t}^{n \alpha} f(t)\right|_{t=0}}{\Gamma(n \alpha+1)},
$$

where $D_{t}^{n \alpha}=\underbrace{D_{t}^{\alpha} \cdot D_{t}^{\alpha} \cdots D_{t}^{\alpha}}_{n \text {-times }}, n=0,1,2 \ldots$

Proof. First of all, notice that if we put $t=0$ into (5), it yields

$$
c_{0}=f(0)=\frac{\left.D_{t}^{0 \alpha} f(t)\right|_{t=0}}{\Gamma(0 \alpha+1)} \text {. }
$$

Applying the operator $D_{t}^{\alpha}$ one time on (4),

$$
c_{1}=\frac{\left.D_{t}^{\alpha} f(t)\right|_{t=0}}{\Gamma(\alpha+1)} \text {. }
$$

Again, by applying the operator $D_{t}^{\alpha}$ two times on (4),

$$
c_{2}=\frac{\left.D_{t}^{2 \alpha} f(t)\right|_{t=0}}{\Gamma(2 \alpha+1)} \text {. }
$$

Analogously

$$
c_{n}=\frac{\left.D_{t}^{n \alpha} f(t)\right|_{t=0}}{\Gamma(n \alpha+1)}, \quad n=0,1,2, \ldots
$$

This completes the proof.

\section{Algorithm of RPS Method for Coupled Systems}

In this section, we consider the following system:

$$
\begin{aligned}
& D_{t}^{p \alpha} u+F(x, t)=0, \\
& D_{t}^{q \alpha} v+G(x, t)=0, \\
& D_{t}^{r \alpha} w+H(x, t)=0
\end{aligned}
$$

with initial values

$$
\begin{gathered}
\left.D_{t}^{i \alpha} u(x, t)\right|_{t=0}=a_{i}(x), \quad i=0,1,2, \ldots, p-1, \\
\left.D_{t}^{j \alpha} v(x, t)\right|_{t=0}=b_{j}(x), \quad j=0,1,2, \ldots, q-1, \\
\left.D_{t}^{k \alpha} w(x, t)\right|_{t=0}=c_{k}(x), \quad k=0,1,2, \ldots, r-1
\end{gathered}
$$

as a generalized illustration for the main idea of RPS method, where the symbol $D_{t}^{(\cdot) \alpha}$ represents the $(\cdot)$ th fractional derivative in the Caputo sense, $\max \{(p-1) / p,(q-1) / q,(r-$ $1) / r\}<\alpha \leq 1(p, q, r \in \mathbb{N})$, the functions $u, v, w, F, G, H$ are analytic at $t=0$, and the initial functions $a_{i}, b_{j}$, and $c_{k}$ are infinitely many times differentiable for all $i=0,1,2, \ldots, p$; $j=0,1,2, \ldots, q$ and $k=0,1,2, \ldots, r$. 
Since $u, v, w, F, G, H$ are analytic at $t=0$, then they can be expanded in the form of fractional power series at $t=0$ as follows:

$$
\begin{aligned}
& u(x, t)=\sum_{i=0}^{\infty} \frac{u_{i}(x)}{\Gamma(i \alpha+1)} t^{i \alpha}, \\
& F(x, t)=\sum_{i=0}^{\infty} \frac{f_{i}(x)}{\Gamma(i \alpha+1)} t^{i \alpha} ; \\
& v(x, t)=\sum_{j=0}^{\infty} \frac{v_{j}(x)}{\Gamma(j \alpha+1)} t^{j \alpha}, \\
& G(x, t)=\sum_{j=0}^{\infty} \frac{g_{j}(x)}{\Gamma(j \alpha+1)} t^{j \alpha} ; \\
& w(x, t)=\sum_{k=0}^{\infty} \frac{w_{k}(x)}{\Gamma(k \alpha+1)} t^{k \alpha}, \\
& H(x, t)=\sum_{k=0}^{\infty} \frac{h_{k}(x)}{\Gamma(k \alpha+1)} t^{k \alpha},
\end{aligned}
$$

where $x \in \mathbb{R}, t \in(-R, R), R$ is the minimum convergence radius of functions $u(x, t), v(x, t), w(x, t), F(x, t), G(x$, $t), H(x, t)$, and

$$
\begin{aligned}
& a_{i}(x)=\left.D_{t}^{i \alpha} u(x, t)\right|_{t=0}, \\
& f_{i}(x)=\left.D_{t}^{i \alpha} F(x, t)\right|_{t=0}, \\
& i=0,1,2, \ldots \text {; } \\
& b_{j}(x)=\left.D_{t}^{j \alpha} u(x, t)\right|_{t=0}, \\
& g_{j}(x)=\left.D_{t}^{j \alpha} G(x, t)\right|_{t=0}, \\
& j=0,1,2, \ldots ; \\
& c_{k}(x)=\left.D_{t}^{k \alpha} u(x, t)\right|_{t=0}, \\
& h_{k}(x)=\left.D_{t}^{k \alpha} H(x, t)\right|_{t=0} \text {, } \\
& k=0,1,2, \ldots .
\end{aligned}
$$

According to the initial conditions,

$$
\begin{gathered}
u_{i}(x)=a_{i}(x), \quad i=0,1,2, \ldots, p-1 \\
v_{j}(x)=b_{j}(x), \quad j=0,1,2, \ldots, q-1 \\
w_{k}(x)=c_{k}(x), \quad k=0,1,2, \ldots, r-1 .
\end{gathered}
$$

Thus the initial approximation of the solution $u, v, w$ is as follows:

$$
\begin{aligned}
u^{\text {init }}(x, t) & =\sum_{i=0}^{p-1} \frac{u_{i}(x)}{\Gamma(i \alpha+1)} t^{i \alpha}, \\
v^{\text {init }}(x, t) & =\sum_{j=0}^{q-1} \frac{v_{j}(x)}{\Gamma(j \alpha+1)} t^{j \alpha}, \\
w^{\text {init }}(x, t) & =\sum_{k=0}^{r-1} \frac{w_{k}(x)}{\Gamma(k \alpha+1)} t^{k \alpha} .
\end{aligned}
$$

Then we calculate the coefficients $u_{i}(x), v_{j}(x)$, and $w_{k}(x)$ for $i=p, p+1, \ldots ; j=q, q+1, \ldots ; k=r, r+1, \ldots$. Firstly, some symbols are given as follows:

$$
\begin{gathered}
\operatorname{Res}_{u}(x, t)=D_{t}^{p \alpha} u+F(x, t), \\
\operatorname{Res}_{u, l}(x, t)=D_{t}^{p \alpha} u_{l}+F(x, t) ; \\
\operatorname{Res}_{v}(x, t)=D_{t}^{q \alpha} u+G(x, t), \\
\operatorname{Res}_{v, m}(x, t)=D_{t}^{q \alpha} v_{m}+F(x, t) ; \\
\operatorname{Res}_{w}(x, t)=D_{t}^{r \alpha} u+H(x, t), \\
\operatorname{Res}_{w, n}(x, t)=D_{t}^{r \alpha} u_{n}+F(x, t),
\end{gathered}
$$

where

$$
\begin{aligned}
u_{l}(x, t) & =\sum_{i=0}^{l} \frac{u_{i}(x)}{\Gamma(i \alpha+1)} t^{i \alpha} \\
& =u^{\text {init }}(x, t)+\sum_{i=p}^{l} \frac{u_{i}(x)}{\Gamma(i \alpha+1)} t^{i \alpha}, \\
v_{m}(x, t) & =\sum_{j=0}^{m} \frac{v_{j}(x)}{\Gamma(j \alpha+1)} t^{j \alpha} \quad l=p, p+1, \ldots ; \\
& =v^{\text {init }}(x, t)+\sum_{i=q}^{m} \frac{v_{i}(x)}{\Gamma(i \alpha+1)} t^{i \alpha},
\end{aligned}
$$

$$
\begin{aligned}
w_{n}(x, t) & =\sum_{k=0}^{n} \frac{w_{k}(x)}{\Gamma(k \alpha+1)} t^{k \alpha} \\
& =w^{\text {init }}(x, t)+\sum_{i=r}^{n} \frac{w_{i}(x)}{\Gamma(i \alpha+1)} t^{i \alpha},
\end{aligned}
$$


Hence there are two facts:

$\left(\mathbf{F}_{1}\right)$

$$
\begin{aligned}
& \lim _{l \rightarrow \infty} u_{l}(x, t)=u(x, t), \\
& \lim _{m \rightarrow \infty} v_{m}(x, t)=u(x, t), \\
& \lim _{n \rightarrow \infty} w_{n}(x, t)=w(x, t) .
\end{aligned}
$$

$\left(\mathbf{F}_{2}\right)$

$$
\begin{aligned}
& \lim _{l \rightarrow \infty} \operatorname{Res}_{u, l}(x, t)=\operatorname{Res}_{u, \infty}(x, t)=\operatorname{Res}_{u}(x, t) \equiv 0 ; \\
& \lim _{m \rightarrow \infty} \operatorname{Res}_{v, m}(x, t)=\operatorname{Res}_{v, \infty}(x, t)=\operatorname{Res}_{v}(x, t) \equiv 0 ; \\
& \lim _{n \rightarrow \infty} \operatorname{Res}_{w, n}(x, t)=\operatorname{Res}_{w, \infty}(x, t)=\operatorname{Res}_{w}(x, t) \equiv 0 .
\end{aligned}
$$

Furthermore

$$
\begin{aligned}
0 & =\left.D_{t}^{(i-p) \alpha} \operatorname{Res}_{u, \infty}(x, t)\right|_{t=0}=u_{i}(x)+D_{t}^{(i-p) \alpha} F(x, t), \quad i=p, p+1, \ldots \\
& \Longrightarrow u_{i}(x)=f_{i-p}(x) \triangleq a_{i}(x), \quad i=p, p+1, \ldots ; \\
0 & =\left.D_{t}^{(j-q) \alpha} \operatorname{Res}_{v, \infty}(x, t)\right|_{t=0}=v_{j}(x)+D_{t}^{(j-q) \alpha} G(x, t), \quad j=q, q+1, \ldots \\
& \Longrightarrow v_{j}(x)=g_{j-q}(x) \triangleq b_{j}(x), \quad j=q, q+1, \ldots ; \\
0 & =\left.D_{t}^{(k-r) \alpha} \operatorname{Res}_{w, \infty}(x, t)\right|_{t=0}=w_{k}(x)+D_{t}^{(k-q) \alpha} H(x, t), \quad k=r, r+1, \ldots \\
& \Longrightarrow w_{k}(x)=h_{k-p}(x) \triangleq c_{k}(x), \quad k=r, p+1, \ldots
\end{aligned}
$$

Thus the solutions of coupled system (10) are

$$
\begin{aligned}
& u(x, t)=\sum_{i=0}^{\infty} \frac{a_{i}(x)}{\Gamma(i \alpha+1)} t^{n \alpha}, \\
& v(x, t)=\sum_{j=0}^{\infty} \frac{b_{j}(x)}{\Gamma(j \alpha+1)} t^{n \alpha}, \\
& w(x, t)=\sum_{k=0}^{\infty} \frac{c_{k}(x)}{\Gamma(k \alpha+1)} t^{n \alpha} .
\end{aligned}
$$

Remark 8. If

$$
\begin{aligned}
& F(x, t)=f(x, t, u, v, w), \\
& G(x, t)=g(x, t, u, v, w), \\
& H(x, t)=h(x, t, u, v, w)
\end{aligned}
$$

and even $F, G, H$ include the term of space fractional derivative and the term of time-fractional derivative whose order is less than the order of the system, then RPS method is also effective in calculating the asymptotic solutions for coupled system (10). In fact, F, G, $H$ could be expanded in the form of fractional power series about time variable $t$ at the initial time $t_{0}$, and facts $\left(\mathbf{F}_{1}\right)$ and $\left(\mathbf{F}_{2}\right)$ are reasonable as well; thus the coefficients appearing in the asymptotic solutions could be obtained successfully.

\section{Application of RPS Method to Time-Space Fractional Coupled Systems}

4.1. The Time-Space Fractional Coupled KdV System. KdV equation plays an important role in nonlinear evolution equation for its wide application in physics and engineering. Coupled KdV system was introduced by Hirota and Satsuma [25] to describe the iterations of water waves and they claimed that the system exits a soliton solution. In [26], Fan and Zhang got several kinds of solutions by an improved homogeneous method. In [20], Bhrawy et al. reduced the time-fractional coupled $\mathrm{KdV}$ equations into a problem consisting of a system of algebraic equations that greatly simplifies the problem via the shifted Legendre polynomials. The time-fractional coupled $\mathrm{KdV}$ equation is a generalization of the classical coupled $\mathrm{KdV}$ equation and in this subsection we generalize time-fractional coupled KdV system to time-space fractional coupled system (23) and obtain the asymptotic solution using RPS method.

Consider the time-space fractional coupled KdV system:

$$
\begin{array}{r}
D_{t}^{\alpha} u-a D_{x}^{3 \beta} u-6 a u D_{x}^{\gamma} u-2 b v D_{x}^{\delta} v=0, \\
D_{t}^{\alpha} v+D_{x}^{3 \lambda} v+3 u D_{x}^{\tau} v=0
\end{array}
$$

with initial values

$$
\begin{aligned}
& u(x, 0)=a_{0}(x), \\
& v(x, 0)=b_{0}(x),
\end{aligned}
$$

where $0<\alpha, \gamma, \delta, \tau \leq 1,2 / 3<\beta, \lambda \leq 1, u=u(x, t), v=$ $v(x, t),(x, t) \in \mathbb{R} \times \mathbb{R}$.

If $u(x, t)$ and $v(x, t)$ are analytic at $t=0$, then they can be expanded in the form of fractional power series 


$$
\begin{aligned}
u(x, t) & =\sum_{i=0}^{\infty} \frac{u_{i}(x)}{\Gamma(i \alpha+1)} t^{i \alpha}, \\
v(x, t) & =\sum_{j=0}^{\infty} \frac{v_{j}(x)}{\Gamma(j \alpha+1)} t^{j \alpha}, \\
F(x, t) & =\sum_{n=0}^{\infty} \frac{t^{n \alpha}}{\Gamma(n \alpha+1)}\left\{-a D_{x}^{3 \beta} u_{n}(x)-\sum_{s=0}^{n} \frac{\Gamma(n \alpha+1)}{\Gamma(s \alpha+1) \Gamma((n-s) \alpha+1)}\left(6 a u_{s}(x) D_{x}^{\gamma} u_{n-s}(x)+2 b v_{s}(x) D_{x}^{\delta} v_{n-s}(x)\right)\right\}, \\
G(x, t) & =\sum_{n=0}^{\infty} \frac{t^{n \alpha}}{\Gamma(n \alpha+1)}\left\{D_{x}^{3 \lambda} v_{n}+\sum_{s=0}^{n} \frac{\Gamma(n \alpha+1)}{\Gamma(s \alpha+1) \Gamma((n-s) \alpha+1)} \cdot 3 u_{s}(x) D_{x}^{\tau} v_{i-1-s}(x)\right\} .
\end{aligned}
$$

Under the initial conditions,

$$
\begin{aligned}
& u_{0}(x)=a_{0}(x), \\
& v_{0}(x)=b_{0}(x) ;
\end{aligned}
$$

that is, the initial approximate solutions are

$$
\begin{aligned}
& u^{\text {initial }}(x, t)=a_{0}(x), \\
& v^{\text {initial }}(x, t)=b_{0}(x) .
\end{aligned}
$$

Set

$$
\begin{aligned}
\operatorname{Res}_{u}(x, t)= & D_{t}^{\alpha} u-a D_{x}^{3 \beta} u-6 a u D_{x}^{\gamma} u-2 b v D_{x}^{\delta} v, \\
\operatorname{Res}_{v}(x, t)= & D_{t}^{\alpha} v+D_{x}^{3 \lambda} v+3 u D_{x}^{\tau} v, \\
\operatorname{Res}_{u, l}(x, t)= & D_{t}^{\alpha} u_{l}-a D_{x}^{3 \beta} u-6 a u_{l} D_{x}^{\gamma} u_{l} \\
& -2 b v_{m} D_{x}^{\delta} v_{m}, \\
\operatorname{Res}_{v, m}(x, t)= & D_{t}^{\alpha} v_{m}+D_{x}^{3 \lambda} v_{m}+3 u_{l} D_{x}^{\tau} v_{m},
\end{aligned}
$$

where

$$
\begin{gathered}
u_{l}(x, t)=a_{0}(x)+\sum_{i=1}^{l} \frac{u_{i}(x)}{\Gamma(i \alpha)+1} t^{i \alpha}, \\
v_{m}(x, t)=b_{0}(x)+\sum_{j=1}^{m} \frac{v_{j}(x)}{\Gamma(j \alpha)+1} t^{j \alpha} .
\end{gathered}
$$

Using RPS method

$$
\begin{aligned}
u_{i}(x) & \\
= & a D_{x}^{3 \beta} u_{i-1}(x)+\sum_{s=0}^{i-1} \frac{\Gamma((i-1) \alpha+1)}{\Gamma(s \alpha+1) \Gamma((i-1-s) \alpha+1)} \\
& \cdot\left\{6 a u_{s}(x) D_{x}^{\gamma} u_{i-1-s}(x)+2 b v_{s}(x) D_{x}^{\delta} v_{i-1-s}(x)\right\} \\
\triangleq & a_{i}(x), \quad i=1,2, \ldots,
\end{aligned}
$$

$$
\begin{aligned}
v_{j}(x) & \\
= & -D_{x}^{3 \lambda} v_{j-1}(x) \\
& \quad-\sum_{s=0}^{j-1} \frac{\Gamma((j-1) \alpha+1)}{\Gamma(s \alpha+1) \Gamma((j-1-s) \alpha+1)} \\
& \cdot 3 u_{s}(x) D_{x}^{\tau} v_{j-1-s}(x) \triangleq b_{j}(x), \quad j=1,2, \ldots
\end{aligned}
$$

Thus the fractional power series solutions of coupled system (23) are

$$
\begin{aligned}
& u(x, t)=\sum_{i=0}^{\infty} \frac{a_{i}(x)}{\Gamma(i \alpha+1)} t^{i \alpha}, \\
& v(x, t)=\sum_{j=0}^{\infty} \frac{b_{j}(x)}{\Gamma(j \alpha+1)} t^{j \alpha} .
\end{aligned}
$$

4.2. The Time-Space Fractional Coupled KdV System of Generalized Hirota-Satsuma Type. In this subsection, we consider the time-space fractional coupled KdV system of generalized Hirota-Satsuma type

$$
\begin{aligned}
D_{t}^{\alpha} u-\frac{1}{2} D_{x}^{3 \beta} u+3 u D_{x}^{\gamma} u-3 D_{x}^{\delta}(v w) & =0, \\
D_{t}^{\alpha} v+D_{x}^{3 \lambda} v-3 u D_{x}^{\tau} v & =0, \\
D_{t}^{\alpha} w+D_{x}^{3 \theta} w-3 u D_{x}^{\sigma} w & =0, \\
u(x, 0) & =a_{0}(x), \\
v(x, 0) & =b_{0}(x), \\
w(x, 0) & =c_{0}(x),
\end{aligned}
$$

where $0<\alpha, \gamma, \delta, \lambda, \tau \leq 1,2 / 3<\beta, \sigma, \theta \leq 1, u=$ $u(x, t), v=v(x, t), w=w(x, t),(x, t) \in \mathbb{R} \times \mathbb{R}$. The equation describes an interaction of two long waves with different dispersion relations. 
If $u, v, w$ are analytic at $t=0$, then $u, v, w$ can be written as the form of fractional power series

$$
\begin{aligned}
& u(x, t)=\sum_{i=0}^{\infty} \frac{u_{i}(x)}{\Gamma(i \alpha+1)} t^{i \alpha}, \\
& v(x, t)=\sum_{j=0}^{\infty} \frac{v_{j}(x)}{\Gamma(j \alpha+1)} t^{j \alpha}, \\
& w(x, t)=\sum_{k=0}^{\infty} \frac{w_{k}(x)}{\Gamma(k \alpha+1)} t^{k \alpha}, \\
& F(x, t)=-\frac{1}{2} D_{x}^{3 \beta} u+3 u D_{x}^{\gamma} u-3 D_{x}^{\delta}(v w) \\
& =\sum_{n=0}^{\infty} \frac{t^{n \alpha}}{\Gamma(n \alpha+1)} \cdot\left\{-\frac{1}{2} D_{x}^{3 \beta} u_{n}(x)\right. \\
& +3 \sum_{s=0}^{n} \frac{\Gamma(n \alpha+1)}{\Gamma(s \alpha+1) \Gamma((n-s) \alpha+1)}\left(u_{s}(x)\right. \\
& \left.\left.\cdot D_{x}^{\gamma} u_{n-s}(x)-v_{n}(x) w_{n-s}(x)\right)\right\} \text {, } \\
& G(x, t)=D_{x}^{3 \lambda} v-3 u D_{x}^{\tau} v=\sum_{n=0}^{\infty} \frac{t^{n \alpha}}{\Gamma(n \alpha+1)} \\
& \cdot\left\{D_{x}^{3 \lambda} v_{n}(x)-3 \sum_{s=0}^{n} \frac{\Gamma(n \alpha+1)}{\Gamma(s \alpha+1) \Gamma((n-s) \alpha+1)}\right. \\
& \left.\cdot u_{s}(x) D_{x}^{\tau} v_{n-s}(x)\right\} \text {, } \\
& H(x, t)=D_{x}^{3 \theta} w-3 u D_{x}^{\sigma} w=\sum_{n=0}^{\infty} \frac{t^{n \alpha}}{\Gamma(n \alpha+1)} \\
& \cdot\left\{D_{x}^{3 \theta} w_{n}(x)-3 \sum_{s=0}^{n} \frac{\Gamma(n \alpha+1)}{\Gamma(s \alpha+1) \Gamma((n-s) \alpha+1)}\right. \\
& \left.\cdot u_{s}(x) D_{x}^{\sigma} w_{n-s}(x)\right\} \text {. }
\end{aligned}
$$

With the initial values

$$
\begin{gathered}
u_{0}(x)=a_{0}(x), \\
v_{0}(x)=b_{0}(x), \\
w_{0}(x)=c_{0}(x),
\end{gathered}
$$

the initial approximate solutions are

$$
\begin{gathered}
u^{\text {initial }}(x, t)=a_{0}(x), \\
v^{\text {initial }}(x, t)=b_{0}(x), \\
w^{\text {initial }}(x, t)=c_{0}(x) .
\end{gathered}
$$

Set

$$
\begin{aligned}
\operatorname{Res}_{u}(x, t)= & D_{t}^{\alpha} u-\frac{1}{2} D_{x}^{3 \beta} u+3 u D_{x}^{\gamma} u-3 D_{x}^{\delta}(v w) \\
= & 0, \\
\operatorname{Res}_{v}(x, t)= & D_{t}^{\alpha} v+D_{x}^{3 \lambda} v-3 u D_{x}^{\tau} v=0, \\
\operatorname{Res}_{w}(x, t)= & D_{t}^{\alpha} w+D_{x}^{3 \theta} w-3 u D_{x}^{\sigma} w=0, \\
\operatorname{Res}_{u, l}(x, t)= & D_{t}^{\alpha} u_{l}-\frac{1}{2} D_{x}^{3 \beta} u_{l}+3 u_{l} D_{x}^{\gamma} u_{l} \\
& -3 D_{x}^{\delta}\left(v_{m} w_{n}\right), \\
\operatorname{Res}_{v, m}(x, t)= & D_{t}^{\alpha} v_{m}+D_{x}^{3 \lambda} v-3 u_{l} D_{x}^{\tau} v_{m}, \\
\operatorname{Res}_{w, n}(x, t)= & D_{t}^{\alpha} w_{n}+D_{x}^{3 \theta} w_{n}-3 u_{l} D_{x}^{\sigma} w_{n},
\end{aligned}
$$

where

$$
\begin{aligned}
& u_{l}(x, t)=a_{0}(x)+\sum_{i=1}^{l} \frac{u_{i}(x)}{\Gamma(i \alpha+1)} t^{\alpha}, \\
& v_{m}(x, t)=b_{0}(x)+\sum_{j=1}^{m} \frac{v_{i}(x)}{\Gamma(j \alpha+1)}, \\
& w_{n}(x, t)=c_{0}(x)+\sum_{k=1}^{n} \frac{w_{k}(x)}{\Gamma(k \alpha+1)} t^{k \alpha},
\end{aligned}
$$

with the results of RPS method:

$$
\begin{aligned}
& u_{i}(x)=\frac{1}{2} D_{x}^{3 \beta} u_{i-1}(x) \\
& -3 \sum_{s=0}^{i-1} \frac{\Gamma((i-1) \alpha+1)}{\Gamma(s \alpha+1) \Gamma((i-1-s) \alpha+1)} \\
& \cdot\left\{u_{s}(x) D_{x}^{\gamma} u_{i-1-s}(x)-D_{x}^{\delta}\left(v_{s}(x) w_{i-1-s}(x)\right)\right\} \\
& \triangleq a_{i}(x), \quad i=1,2, \ldots, \\
& v_{j}(x)=-D_{x}^{3 \sigma} v_{j-1}(x) \\
& +3 \sum_{s=0}^{j-1} \frac{\Gamma((j-1) \alpha+1)}{\Gamma(j \alpha+1) \Gamma((j-1-s) \alpha+1)} u_{s}(x) \\
& \cdot D_{x}^{\lambda} v_{j-1-s}(x) \triangleq b_{j}(x), \quad j=1,2, \ldots, \\
& w_{k}(x)=-D_{x}^{3 \theta} w_{k-1}(x) \\
& +3 \sum_{s=0}^{k-1} \frac{\Gamma((k-1) \alpha+1)}{\Gamma(j \alpha+1) \Gamma((k-1-s) \alpha+1)} u_{s}(x) \\
& \quad \cdot D_{x}^{\tau} w_{k-1-j}(x) \triangleq c_{k}(x), \quad k=1,2, \ldots
\end{aligned}
$$


So the fractional power series solutions of coupled system (32) are

$$
\begin{aligned}
& u(x, t)=\sum_{i=0}^{\infty} \frac{a_{i}(x)}{\Gamma(i \alpha+1)} t^{i \alpha}, \\
& v(x, t)=\sum_{j=0}^{\infty} \frac{b_{j}(x)}{\Gamma(j \alpha+1)} t^{j \alpha}, \\
& w(x, t)=\sum_{k=0}^{\infty} \frac{c_{k}(x)}{\Gamma(k \alpha+1)} t^{k \alpha} .
\end{aligned}
$$

4.3. The Time-Space Fractional Coupled Whitham-Broer-Kaup (WBK) System. Whitham [27], Broer [28], and Kaup [29] obtained nonlinear WBK system. In [30], Rashidi et al. obtained the approximate traveling wave solutions of the coupled WBK system in shallow water using homotopy analysis method. In [31], Kadem and Baleanu applied the homotopy perturbation method to find an analytical approximate solution for the coupled WBK system. In this subsection, we consider the time-space fractional coupled WBK system and construct the approximate solution by RPS method.

Consider the time-space fractional coupled WBK system:

$$
\begin{aligned}
D_{t}^{\alpha} u+u D_{x}^{\beta} u+D_{x}^{\gamma} v+a D_{x}^{2 \delta} u & =0, \\
D_{t}^{\alpha} v+D_{x}^{\lambda}(u v)-a D_{x}^{2 \tau} v+b D_{x}^{3 \theta} u & =0, \\
u(x, 0) & =a_{0}(x), \\
v(x, 0) & =b_{0}(x),
\end{aligned}
$$

where $0<\alpha, \sigma, \tau, \lambda \leq 1,1 / 2<\delta, \eta \leq 1,2 / 3<\theta \leq$ $1,(x, t) \in \mathbb{R} \times \mathbb{R}, a, b \in \mathbb{R}$ represent different dispersive power, $u=u(x, t)$ is the field of horizontal velocity, and $v=v(x, t)$ is the height deviating equilibrium position of liquid. And this is a very good model to describe dispersive wave.

If $u, v$ are analytic at $t=0$, then $u, v$ can be expanded in the form of fractional power series

$$
\begin{aligned}
& u(x, t)=\sum_{i=0}^{\infty} \frac{u_{i}(x)}{\Gamma(i \alpha+1)} t^{i \alpha}, \\
& v(x, t)=\sum_{j=0}^{\infty} \frac{v_{j}(x)}{\Gamma(j \alpha+1)} t^{j \alpha}, \\
& F(x, t)=\sum_{n=0}^{\infty} \frac{t^{n \alpha}}{\Gamma(n \alpha+1)} \\
& \cdot\left\{\sum_{s=0}^{n} \frac{\Gamma(n \alpha+1)}{\Gamma(s \alpha+1) \Gamma((n-s) \alpha+1)} u_{s}(x) D_{x}^{\beta} u_{n-s}(x)\right. \\
&\left.+D_{x}^{\gamma} v_{n}(x)+a D_{x}^{2 \delta} u_{n}(x)\right\},
\end{aligned}
$$

$G(x, t)$

$$
\begin{aligned}
& =\sum_{n=0}^{\infty} \frac{t^{n \alpha}}{\Gamma(n \alpha+1)}\left\{\sum_{s=0}^{n} \frac{\Gamma(n \alpha+1)}{\Gamma(s \alpha+1) \Gamma((n-s) \alpha+1)}\right. \\
& \left.\cdot D_{x}^{\lambda}\left(u_{s}(x) v_{n-s}(x)\right)+a D_{x}^{2 \tau} v_{n}(x)+b D_{x}^{3 \theta} u_{n}(x)\right\} .
\end{aligned}
$$

Under the initial conditions

$$
\begin{aligned}
& u_{0}(x)=a_{0}(x), \\
& v_{0}(x)=b_{0}(x),
\end{aligned}
$$

and the initial approximate solutions are

$$
\begin{aligned}
& u^{\text {initial }}(x, t)=a_{0}(x), \\
& v^{\text {initial }}(x, t)=b_{0}(x, t) .
\end{aligned}
$$

Set

$$
\begin{aligned}
\operatorname{Res}_{u}(x, t)= & D_{t}^{\alpha} u+u D_{x}^{\beta} u+D_{x}^{\gamma} v+a D_{x}^{2 \delta} u, \\
\operatorname{Res}_{v}(x, t)= & D_{t}^{\alpha} v+D_{x}^{\lambda}(u v)-a D_{x}^{2 \tau} v+b D_{x}^{3 \theta} u, \\
\operatorname{Res}_{u, l}(x, t)= & D_{t}^{\alpha} u_{l}+u_{l} D_{x}^{\beta} u_{l}+D_{x}^{\gamma} v_{m}+a D_{x}^{2 \delta} u_{l}, \\
\operatorname{Res}_{v, m}(x, t)= & D_{t}^{\alpha} v_{m}+D_{x}^{\lambda}\left(u_{l} v_{m}\right)-a D_{x}^{2 \tau} v_{m} \\
& +b D_{x}^{3 \theta} u_{l},
\end{aligned}
$$

where

$$
\begin{aligned}
& u_{l}(x, t)=a_{0}(x)+\sum_{i=1}^{l} \frac{u_{i}(x)}{\Gamma(i \alpha+1)} t^{\alpha}, \\
& v_{m}(x, t)=b_{0}(x)+\sum_{j=1} \frac{v_{j}(x)}{\Gamma(j \alpha+1)} t^{j \alpha} .
\end{aligned}
$$

With RPS method

$$
\begin{gathered}
u_{i}(x)=-\sum_{s=0}^{i-1} \frac{\Gamma((i-1) \alpha+1)}{\Gamma(s \alpha+1) \Gamma((i-1-s) \alpha+1)} u_{s}(x) \\
\cdot D_{x}^{\beta} u_{i-1-s}(x)+D_{x}^{\gamma} v_{i-1}(x)+a D_{x}^{2 \delta} u_{i-1}(x) \\
\triangleq a_{i}(x), \quad i=1,2, \ldots, \\
v_{j}(x)=-\sum_{s=0}^{j-1} \frac{\Gamma((j-1) \alpha+1)}{\Gamma(s \alpha+1) \Gamma((j-1-s) \alpha+1)} \\
\cdot D_{x}^{\lambda}\left(u_{s}(x) v_{j-1-s}(x)\right)+a D_{x}^{2 \tau} v_{j-1}(x) \\
-b D_{x}^{3 \theta} u_{j-1}(x) \triangleq b_{j}(x), \quad j=1,2, \ldots
\end{gathered}
$$


So the fractional power series solution of coupled system (40) is

$$
\begin{aligned}
& u(x, t)=\sum_{i=0}^{\infty} \frac{a_{i}(x)}{\Gamma(i \alpha+1)} t^{i \alpha}, \\
& v(x, t)=\sum_{j=0}^{\infty} \frac{b_{j}(x)}{\Gamma(j \alpha+1)} t^{j \alpha} .
\end{aligned}
$$

Remark 9. When $\alpha=\sigma=\tau=\delta=\lambda=\eta=\theta=1, \beta \neq 0$, $\gamma=0,(40)$ reduces to the classical long-wave equations that describe the shallow water wave with diffusion.

Remark 10. When $\alpha=\sigma=\tau=\delta=\lambda=\eta=\theta=1, \beta=0$, $\gamma=1,(40)$ reduces to the variant Boussinesq equation.

\subsection{The Time-Space Fractional Coupled Shallow Water Sys-} tem. Shallow water systems are widely used in predicting hydrodynamics of surface flows such as water flows in rivers, channels, flood plains, and coastal regions. It is well known that the shallow water systems can accurately predict the hydraulic parameters under conditions of slow erosion and low sediment concentration of the time-space fractional coupled shallow water system [32]. In this subsection, consider the time-space fractional coupled shallow water system

$$
\begin{array}{r}
D_{t}^{\alpha} u+u D_{x}^{\beta} u+D_{x}^{\gamma} v+a D_{x}^{2 \delta} u=0, \\
D_{t}^{\alpha} v+v D_{x}^{\lambda} u+u D_{x}^{\tau} v-a D_{x}^{2 \theta} v+b D_{x}^{3 \sigma} u=0,
\end{array}
$$

with initial values

$$
\begin{aligned}
& u(x, 0)=a_{0}(x), \\
& v(x, 0)=b_{0}(x),
\end{aligned}
$$

where $0<\alpha, \beta, \gamma, \lambda, \tau \leq 1,1 / 2<\delta, \theta \leq 1,2 / 3<\sigma \leq 1, u=$ $u(x, t), v=v(x, t),(x, t) \in \mathbb{R} \times \mathbb{R}$.

If $u, v$ are analytic at $t=0$, then $u, v$ can be written as the form of fractional power series:

$$
\begin{aligned}
& u(x, t)=\sum_{i=0}^{\infty} \frac{u_{i}(x)}{\Gamma(i \alpha+1)} t^{i \alpha}, \\
& v(x, t)=\sum_{j=0}^{\infty} \frac{v_{j}(x)}{\Gamma(j \alpha+1)} t^{j \alpha}, \\
& F(x, t)=\sum_{n=0}^{\infty} \frac{t^{n \alpha}}{\Gamma(n \alpha+1)} \\
& \cdot\left\{\sum_{s=0}^{n} \frac{\Gamma(n \alpha+1)}{\Gamma(s \alpha+1) \Gamma((n-s) \alpha+1)} u_{s}(x) D_{x}^{\beta} u_{n-s}(x)\right. \\
&\left.+D_{x}^{\gamma} v_{n}(x)+a D_{x}^{2 \delta} u_{n}(x)\right\},
\end{aligned}
$$

$$
\begin{aligned}
& G(x, t)=\sum_{n=0}^{\infty} \frac{t^{n \alpha}}{\Gamma(n \alpha+1)} \\
& \cdot\left\{\sum_{s=0}^{n} \frac{\Gamma(n \alpha+1)}{\Gamma(s \alpha+1) \Gamma((n-s) \alpha+1)}\right. \\
& \cdot\left(v_{s}(x) D_{x}^{\lambda} u_{n-s}(x)+u_{s}(x) D_{x}^{\tau} v_{n-s}(x)\right) \\
& \left.-a D_{x}^{2 \theta} v_{n}(x)+b D_{x}^{3 \sigma} u_{n}(x)\right\} .
\end{aligned}
$$

With the initial conditions

$$
\begin{aligned}
& u_{0}(x)=a_{0}(x), \\
& v_{0}(x)=b_{0}(x),
\end{aligned}
$$

and the initial approximate solutions are

$$
\begin{gathered}
u^{\text {initial }}(x, t)=a_{0}(x), \\
v^{\text {initial }}(x, t)=b_{0}(x) .
\end{gathered}
$$

Set

$$
\begin{aligned}
& \operatorname{Res}_{u}(x, t)=D_{t}^{\alpha} u+u D_{x}^{\beta} u+D_{x}^{\gamma} v+a D_{x}^{2 \delta} u, \\
& \operatorname{Res}_{v}(x, t) D_{t}^{\alpha} v+v D_{x}^{\lambda} u+u D_{x}^{\tau} v-a D_{x}^{2 \theta} v+b D_{x}^{3 \sigma} u, \\
& \operatorname{Res}_{u, l}(x, t)=D_{t}^{\alpha} u_{l}+u_{l} D_{x}^{\beta} u_{l}+D_{x}^{\gamma} v_{m}+a D_{x}^{2 \delta} u_{l}, \\
& \operatorname{Res}_{v, m}(x, t)=D_{t}^{\alpha} v_{m}+v_{m} D_{x}^{\lambda} u_{l}+u_{l} D_{x}^{\tau} v_{m}-a D_{x}^{2 \theta} v_{m} \\
& \quad+b D_{x}^{3 \sigma} u_{l},
\end{aligned}
$$

where

$$
\begin{gathered}
u_{l}(x, t)=a_{0}(x)+\sum_{i=1}^{l} \frac{u_{i}(x)}{\Gamma(i \alpha+1)} t^{i \alpha}, \\
v_{m}(x, t)=b_{0}(x)+\sum_{j=1}^{m} \frac{v_{j}(x)}{\Gamma(j \alpha+1)} t^{j \alpha} .
\end{gathered}
$$

Using the RPS method

$$
\begin{gathered}
u_{i}(x)=-\sum_{s=0}^{i-1} \frac{\Gamma((i-1) \alpha+1)}{\Gamma(s \alpha+1) \Gamma((i-1-s) \alpha+1)} u_{s}(x) \\
\cdot D_{x}^{\beta} u_{i-1-s}(x)-D_{x}^{\gamma} v_{i-1}(x)+a D_{x}^{2 \delta} u_{i-1}(x) \\
\triangleq a_{i}(x), \quad i=1,2, \ldots, \\
v_{j}(x)=-\sum_{s=0}^{i-1} \frac{\Gamma((i-1) \alpha+1)}{\Gamma(s \alpha+1) \Gamma((i-1-s) \alpha+1)}\left(v_{s}(x)\right. \\
\left.\cdot D_{x}^{\lambda} u_{j-1-s}+u_{s}(x) D_{x}^{\tau} v_{j-1-s}(x)\right)+a D_{x}^{2 \theta} v_{j-1}(x) \\
-b D_{x}^{3 \sigma} u_{i-1}(x) \triangleq b_{j}(x), \quad j=1,2, \ldots
\end{gathered}
$$


So the solutions of coupled system (48) are

$$
\begin{aligned}
& u(x, t)=\sum_{n=0}^{\infty} \frac{a_{n}(x)}{\Gamma(n \alpha+1)} t^{n \alpha}, \\
& v(x, t)=\sum_{n=0}^{\infty} \frac{b_{n}(x)}{\Gamma(n \alpha+1)} t^{n \alpha} .
\end{aligned}
$$

\section{Concluding Remarks}

This paper introduced a new analytical iterative technique to construct asymptotic solutions to time-space fractional coupled systems, which is based on the general Residual Power Series method. Furthermore, we apply this method to some specific time-space fractional coupled systems to obtain asymptotic solutions with respect to initial values, which shows that this method is efficient and does not require linearization or perturbation.

\section{Conflicts of Interest}

The authors declare that there are no conflicts of interest regarding the publication of this paper.

\section{Acknowledgments}

The researches of Yanni Pang and of Wenjin Li were supported by NSFC Grants 11626043 and 71302039, respectively.

\section{References}

[1] G. Leibniz, "Letter from hanover, Germany, deptember 30, 1695 to ga lhospital," JLeibnizen Mathematische Schriften, vol. 2, no. 301-302, 1849.

[2] K. S. Miller and B. Ross, An Introduction to the Fractional Calculus and Fractional Differential Equations, A Wiley-Interscience Publication, John Wiley \& Sons, New York, NY, USA, 1993.

[3] K. Sakamoto and M. Yamamoto, "Initial value/boundary value problems for fractional diffusion-wave equations and applications to some inverse problems," Journal of Mathematical Analysis and Applications, vol. 382, no. 1, pp. 426-447, 2011.

[4] P. Goswami and R. T. Alqahtani, "Solutions of fractional differential equations by Sumudu transform and variational iteration method," Journal of Nonlinear Science and its Applications, vol. 9, no. 4, pp. 1944-1951, 2016.

[5] V. K. Saraswathy, The numerical solutions of fractional differential equations with fractional Taylor vector [Ph.D. thesis], ProQuest LLC, Mississippi State University, Ann Arbor, MI, USA, 2016.

[6] V. Daftardar-Gejji and H. Jafari, "An iterative method for solving nonlinear functional equations," Journal of Mathematical Analysis and Applications, vol. 316, no. 2, pp. 753-763, 2006.

[7] C. D. Dhaigude and V. R. Nikam, "Solution of fractional partial differential equations using iterative method," Fractional Calculus and Applied Analysis, vol. 15, no. 4, pp. 684-699, 2012.

[8] F. Xu, Y. Gao, and W. Zhang, "Construction of analytic solution for time-fractional Boussinesq equation using iterative method," Advances in Mathematical Physics, vol. 2015, Article ID 506140, 7 pages, 2015.
[9] O. Abu Arqub, Z. Abo-Hammour, R. Al-Badarneh, and S. Momani, "A reliable analytical method for solving higher-order initial value problems," Discrete Dynamics in Nature and Society, vol. 2013, Article ID 673829, 12 pages, 2013.

[10] O. A. Arqub, A. El-Ajou, Z. A. Zhour, and S. Momani, "Multiple solutions of nonlinear boundary value problems of fractional order: a new analytic iterative technique," Entropy, vol. 16, no. 1, pp. 471-493, 2014.

[11] A. El-Ajou, O. Abu Arqub, and S. Momani, "Approximate analytical solution of the nonlinear fractional KdV-Burgers equation: a new iterative algorithm," Journal of Computational Physics, vol. 293, pp. 81-95, 2015.

[12] F. Xu, Y. Gao, X. Yang, and H. Zhang, "Construction of fractional power series solutions to fractional Boussinesq equations using residual power series method," Mathematical Problems in Engineering, vol. 2016, Article ID 5492535, 15 pages, 2016.

[13] W. Yin, F. Xu, W. Zhang, and Y. Gao, "Asymptotic expansion of the solutions to time-space fractional Kuramoto-Sivashinsky equations," Advances in Mathematical Physics, vol. 2016, 9 pages, 2016.

[14] S. S. Ezz-Eldien, "New quadrature approach based on operational matrix for solving a class of fractional variational problems," Journal of Computational Physics, vol. 317, pp. 362381, 2016.

[15] A. H. Bhrawy and S. S. Ezz-Eldien, "A new Legendre operational technique for delay fractional optimal control problems," Calcolo, vol. 53, no. 4, pp. 521-543, 2016.

[16] S. S. Ezz-Eldien, A. H. Bhrawy, and A. A. El-Kalaawy, "Direct numerical method for isoperimetric fractional variational problems based on operational matrix," Journal of Vibration and Control, article 107754631770034, 2017.

[17] C.-z. Bai and J.-x. Fang, "The existence of a positive solution for a singular coupled system of nonlinear fractional differential equations," Applied Mathematics and Computation, vol. 150, no. 3, pp. 611-621, 2004.

[18] Y. Chen and H.-L. An, "Numerical solutions of coupled Burgers equations with time- and space-fractional derivatives," Applied Mathematics and Computation, vol. 200, no. 1, pp. 87-95, 2008.

[19] A. Yldrm and A. Kelleci, "Homotopy perturbation method for numerical solutions of coupled Burgers equations with time- and space-fractional derivatives," International Journal of Numerical Methods for Heat \& Fluid Flow, vol. 20, no. 8, pp. 897909, 2010.

[20] A. H. Bhrawy, E. H. Doha, S. S. Ezz-Eldien, and M. A. Abdelkawy, "A numerical technique based on the shifted Legendre polynomials for solving the time-fractional coupled KdV equations," Calcolo, vol. 53, no. 1, pp. 1-17, 2016.

[21] S. S. Ray, "Soliton solutions for time fractional coupled modified $\mathrm{KdV}$ equations using new coupled fractional reduced differential transform method," Journal of Mathematical Chemistry, vol. 51, no. 8, pp. 2214-2229, 2013.

[22] A. El-Ajou, O. Abu Arqub, Z. Al Zhour, and S. Momani, "New results on fractional power series: theories and applications," Entropy, vol. 15, no. 12, pp. 5305-5323, 2013.

[23] A. A. Kilbas, H. M. Srivastava, and J. J. Trujillo, Theory and Applications of Fractional Differential Equations, vol. 204 of North-Holland Mathematics Studies, Elsevier Science, Amsterdam, Netherlands, 2006.

[24] B. Ross, "A brief history and exposition of the fundamental theory of fractional calculus," in Fractional Calculus and Its Applications, vol. 457 of Lecture Notes in Mathematics, pp. 1-36, Springer Berlin Heidelberg, Berlin, Germany, 1975. 
[25] R. Hirota and J. Satsuma, "Soliton solutions of a coupled Korteweg-de Vries equation," Physics Letters. A, vol. 85, no. 89, pp. 407-408, 1981.

[26] E. Fan and H. Zhang, "New exact solutions to a solutions to a system of coupled KdV equations," Physics Letters. A, vol. 245, no. 5, pp. 389-392, 1998.

[27] G. B. Whitham, "Variational methods and applications to water waves," in Hyperbolic Equations and Waves (Rencontres, Battelle RES. Inst., Seattle, Wash., 1968), pp. 153-172, Springer, Berlin, Germany, 1970.

[28] L. J. Broer, "Approximate equations for long water waves," Applied Scientific Research, vol. 31, no. 5, pp. 377-395, 1975.

[29] D. J. Kaup, "A higher-order water-wave equation and the method for solving it," Progress of Theoretical Physics, vol. 54, no. 2, pp. 396-408, 1975.

[30] M. M. Rashidi, D. D. Ganji, and S. Dinarvand, "Approximate traveling wave solutions of coupled Whitham-Broer-Kaup shallow water equations by homotopy analysis method," Differential Equations and Nonlinear Mechanics, vol. 2008, Article ID 243459, 8 pages, 2008.

[31] A. Kadem and D. Baleanu, "On fractional coupled WhithamBroer-Kaup equations," Romanian Journal of Physics, vol. 56, no. 5-6, pp. 629-635, 2011.

[32] X. Liu, A. Mohammadian, A. Kurganov, and J. A. Infante Sedano, "Well-balanced central-upwind scheme for a fully coupled shallow water system modeling flows over erodible bed," Journal of Computational Physics, vol. 300, pp. 202-218, 2015. 


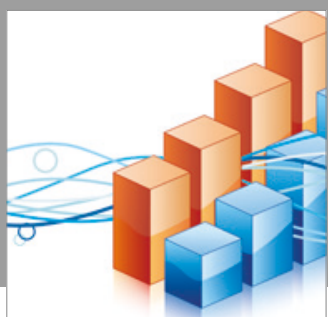

Advances in

Operations Research

vatersals

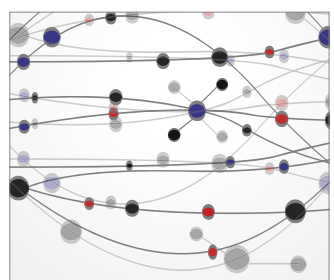

\section{The Scientific} World Journal
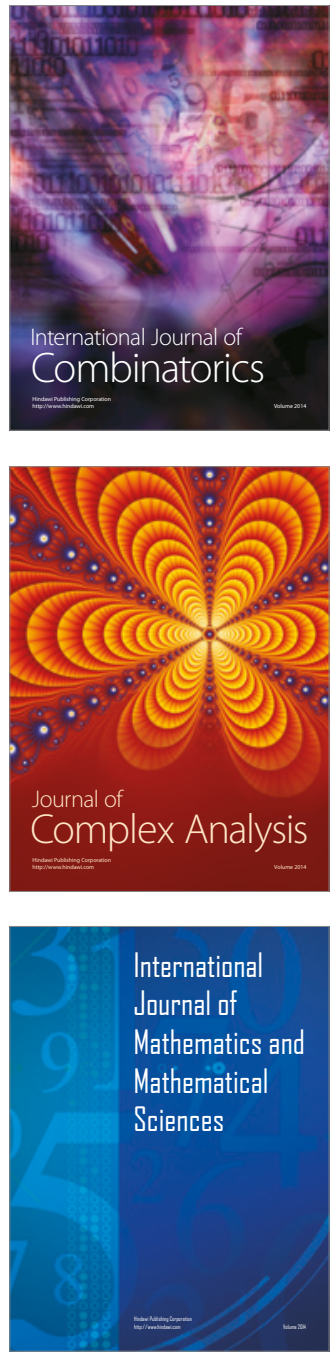
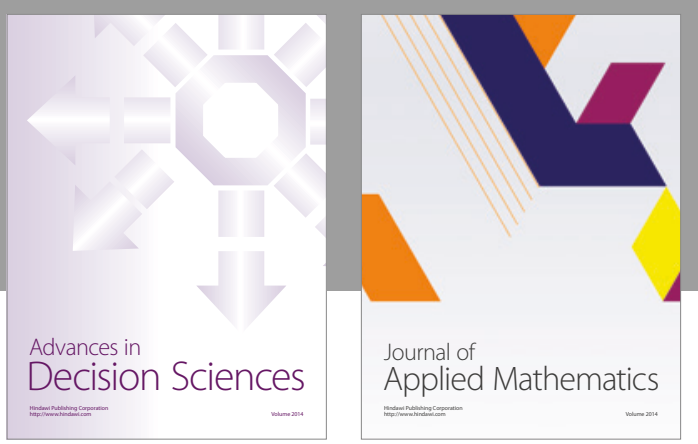

Algebra

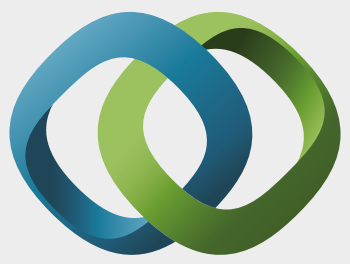

\section{Hindawi}

Submit your manuscripts at

https://www.hindawi.com
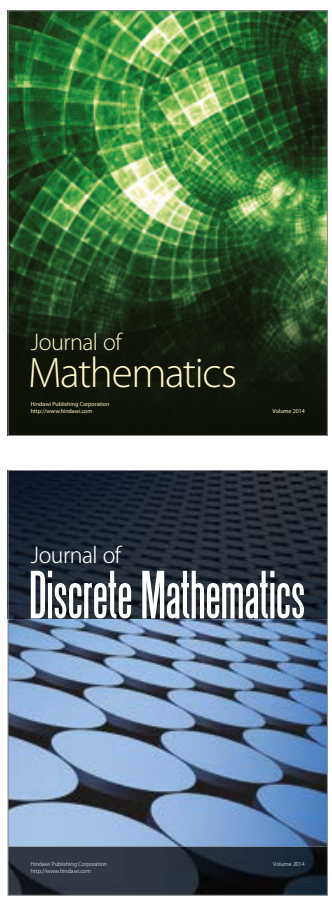

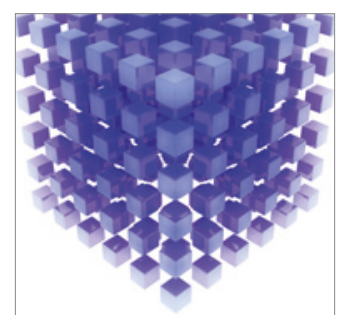

Mathematical Problems in Engineering
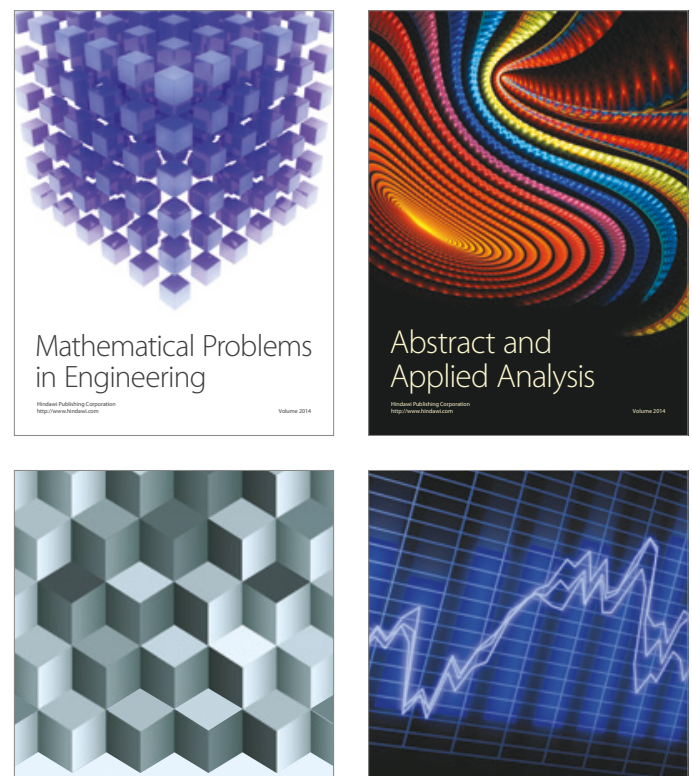

Journal of

Function Spaces

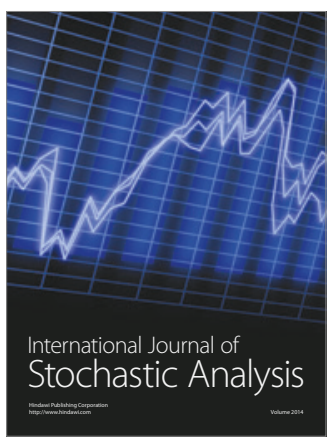

Probability and Statistics
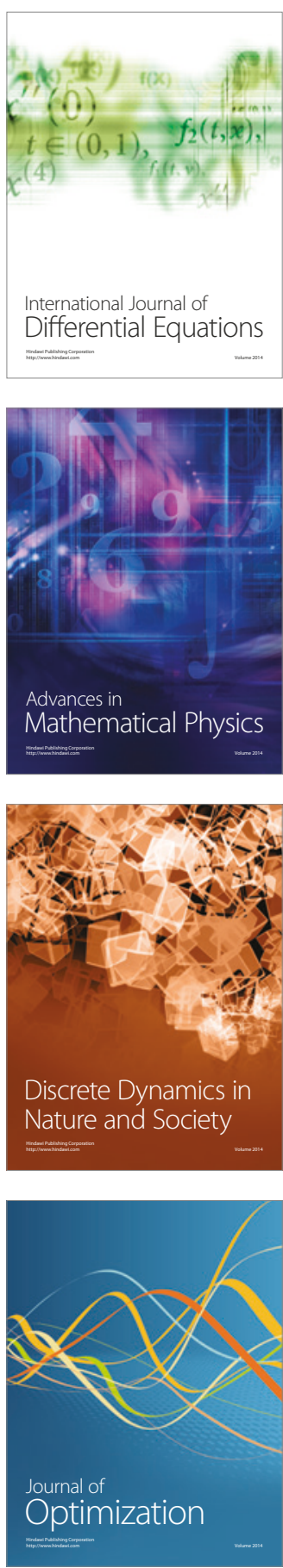